\title{
The Widom insertion method and ordering in small hard-sphere systems
}

\author{
ROEL P. A. DULLENS*, DIRK G. A. L. AARTS, WILLEM K. KEGEL \\ and HENK N. W. LEKKERKERKER \\ Van't Hoff Laboratory for Physical and Colloid Chemistry, Debye Institute, Utrecht University, \\ Padualaan 8, $3584 \mathrm{CH}$ Utrecht, The Netherlands
}

(Received 2 May 2005; in final form 10 June 2005)

\begin{abstract}
We study a small system of spherically confined hard spheres using the Widom particle insertion method as an indicator for the ordering transition. By Monte Carlo simulations we determine the insertion probability as a function of the number of particles and the box size. We find an increase of the insertion probability upon increasing density, which becomes more pronounced as the box size decreases. The increase of the insertion probability corresponds to an increase of the order, which is confirmed by a local bond-order analysis and the simulation snapshots. The ordered structures can be described as defective solids.
\end{abstract}

\section{Introduction}

Ben Widom has provided us with many deep and elegant results in the statistical mechanics of liquids and interfaces. One of these is [1], which is referred to by Ben Widom as the potential distribution function theory [2] and more commonly known as the Widom particle insertion method. The basic expression of this principle is

$$
\frac{\rho}{z}=\left\langle\exp \left[\frac{-\psi}{k_{\mathrm{B}} T}\right]\right\rangle
$$

where $\rho=N / V$ is the particle number density, $z$ is the thermodynamic activity, $\psi$ is the interaction energy of a test particle in a sea of $N-1$ particles and \langle\rangle refers to the canonical average. The number of particles is denoted by $N$ and $V$ is the volume of the system. In particular, for hard spherical particles equation (1) takes on a simple form:

$$
\frac{\rho}{z}=\frac{V_{0}}{V}
$$

with $V_{0}$ the available volume for the test hard sphere in a sea of $N-1$ hard spheres. Equation (2) is the point of departure to 'measure' the chemical potential with

\footnotetext{
*Corresponding author. Email: r.p.a.dullens@chem.uu.nl
}

computer simulations [3]. Recently, it has been shown that the Widom particle insertion method can also be applied directly to experimental (colloidal) particle configurations obtained by confocal microscopy [4].

Equation (2) can furthermore be used as a convenient indicator for phase transitions in a system of hard particles. Consider the hard-sphere fluid-crystal transition, where the coexisting volume fractions in the fluid and crystal phases are denoted by $\phi_{\mathrm{F}}$ and $\phi_{\mathrm{C}}$ and the thermodynamic activity at coexistence by $z^{*}$. Then clearly it follows from equation (2) that the plot of $V_{0} / V$ versus $\phi(\phi \equiv \rho v$, with $v$ the particle volume) looks as shown in figure 1. Clearly, the hard-sphere phase transition is indicated by the minimum of $V_{0} / V$ at $\phi_{\mathrm{F}}$ and the subsequent increase until $\phi_{\mathrm{C}}$. This behaviour of $V_{0} / V$ as a function of $\phi$ was used to demonstrate the existence of the hard-sphere fluid-crystal transition form, available using volume calculations in small boxes [7, 8]. Although equations (1) and (2) are valid in the thermodynamic limit $N \rightarrow \infty$, increasing insertion probability as a function of the density still proved to be characteristic for ordering, even in such small systems $[7,8]$.

Here we will use this strategy to explore the behaviour of very small systems of hard spheres $(1 \leq N \leq 13)$ in spherical confinement. The spherical geometry introduces no bias towards predefined lattice sites in the form of corners, in contrast to earlier work involving rectangular confinement $[7,8]$. Hard spheres in spherical cavities have attracted some attention the past few years. Density functional theories have been applied 


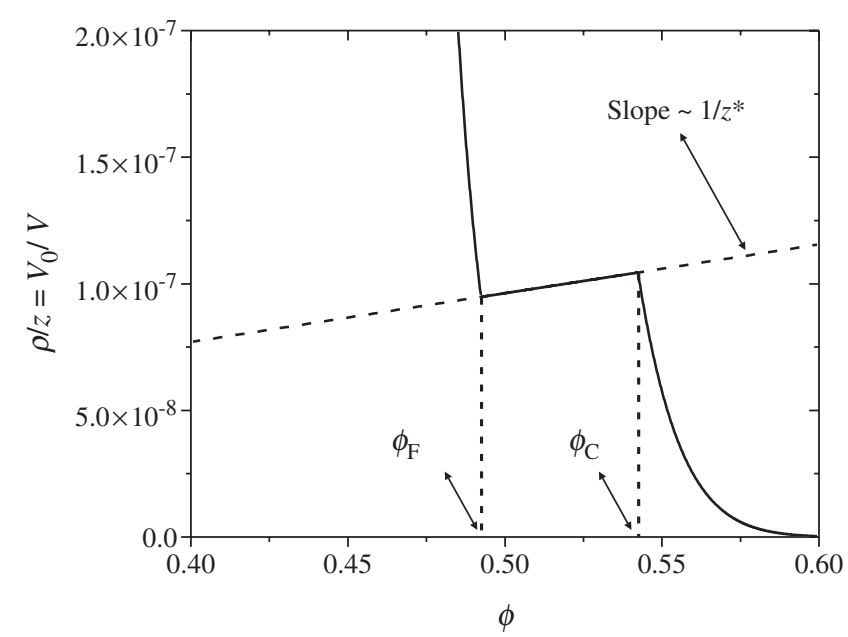

Figure 1. The insertion probability $V_{0} / V$ for hard spheres as a function of the volume fraction $\phi$. The coexisting volume fractions in the fluid and crystal phases are indicated by respectively $\phi_{\mathrm{F}}$ and $\phi_{\mathrm{C}}$. The slope at coexistence is given by the inverse of the thermodynamic activity at coexistence: $1 / z^{*}$. For the calculation of the fluid- and crystal-branches respectively the Carnahan-Starling equation of state [5] and the equation of state of the hard-sphere solid as obtained from computer simulations [6] have been used.

to describe the confined fluid [9-11] and molecular dynamics simulations have been used to address freezing and glass formation of hard spheres in cavities [12]. In this work, we use the Widom particle insertion method to study ordering of hard spheres in a spherical cavity using Monte Carlo simulations. In addition, we show how such systems may be studied experimentally.

\section{Simulation and analysis methods}

We performed standard Monte Carlo simulations in the canonical ensemble [3]. The centres of the hard spheres with diameter $\sigma$ were contained in a spherical simulation box of diameter $D$. A schematic illustration of the geometry of the system is shown in figure 2 . A simulation cycle started with an equilibration of $5 \times 10^{4}$ moves per particle. The step length during equilibration was adjusted such that the Monte Carlo acceptance rate was in the range $0.3-0.5$ for all $N$. The number of particles was varied from 1 to 13 and the reduced diameter $D / \sigma$ from 2.10 to 2.30 . Subsequently, the insertion probability $V_{0} / V$ was computed numerically by $1 \times 10^{5}$ insertion attempts per particle in the simulation box. Typically, the data were averaged over $10^{3}$ simulation cycles.

For the case of 13 particles in a spherical box with $D / \sigma=2.10$, the central sphere is surrounded by 12 neighbouring particles constituting the coordination

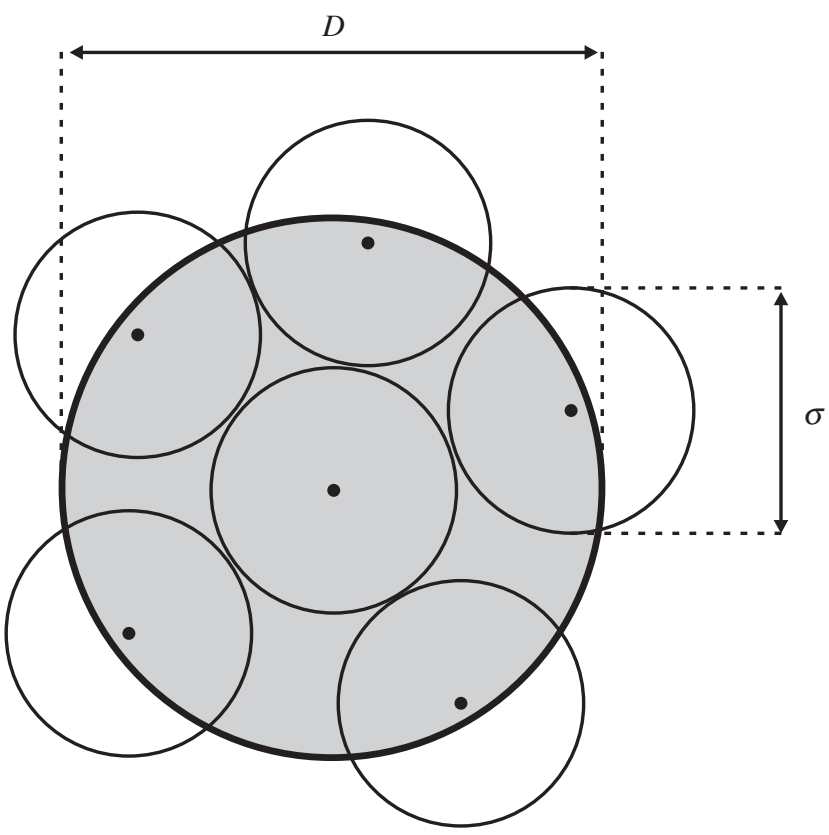

Figure 2. Schematic picture of the geometry of the system. The spherical simulation box with diameter $D$ (in grey) contains the centres (black dots) of the hard spheres (black circles) of diameter $\sigma$.

shell. Starting from a face-centred cubic unit cell, the particles spontaneously form an icosahedral structure after only a small fraction of the equilibration period. To quantify the degree of order, i.e. icosahedral character, the structure of the particles constituting the coordination shell was measured using a local bondorder parameter that is based on spherical harmonics $[13,14]$ :

$$
q_{l m}(i)=\frac{1}{n_{\mathrm{c}}} \sum_{j=1}^{n_{\mathrm{c}}} Y_{l m}\left(\hat{r}_{i j}\right) .
$$

Here, $Y_{l m}$ are the spherical harmonic functions, $n_{\mathrm{c}}$ is the number of nearest neighbours of a hypothetical particle $i$ located at the centre of the simulation box and $\hat{r}_{i j}$ is the unit vector parallel to the bond of the hypothetical particle $i$ with nearest neighbour $j$. If the radial distance of a particle was smaller than $0.25 D / \sigma$, we ignored this particle for the calculation of $q_{6}$. The nearest neighbours were determined using a cut-off distance equal to $1.25 \sigma$. As $q_{l m}$ depend on the reference frame, it is convenient to use the rotationally invariant forms

$$
q_{l}(i)=\left(\frac{4 \pi}{2 l+1} \sum_{m=-l}^{l}\left|q_{l m}(i)\right|^{2}\right)^{1 / 2} .
$$


In this work we only considered the parameter $q_{l}$ for $l=6$, which equals 0.66 for a perfect icosahedral structure (under the absence of thermal motion). Furthermore, we determined the radial number profiles, $n(r)$, where $n(r)$ is the number of particles between $r \pm \Delta r_{\text {bin }} / 2$ with $\Delta r_{\text {bin }}$ the bin size.

\section{Results and discussion}

In figure 3 we show the normalized radial number profiles for $N=11$ and $D / \sigma=2.10$. Clearly, two distinct scenarios are observed. The profile shown in figure 3 (a) shows two peaks indicating the presence of a central particle surrounded by a coordination shell constituted by the remaining spheres. The height ratio of the peaks is 0.1 , which is consistent with one central sphere surrounded by the 10 other particles. As there is one

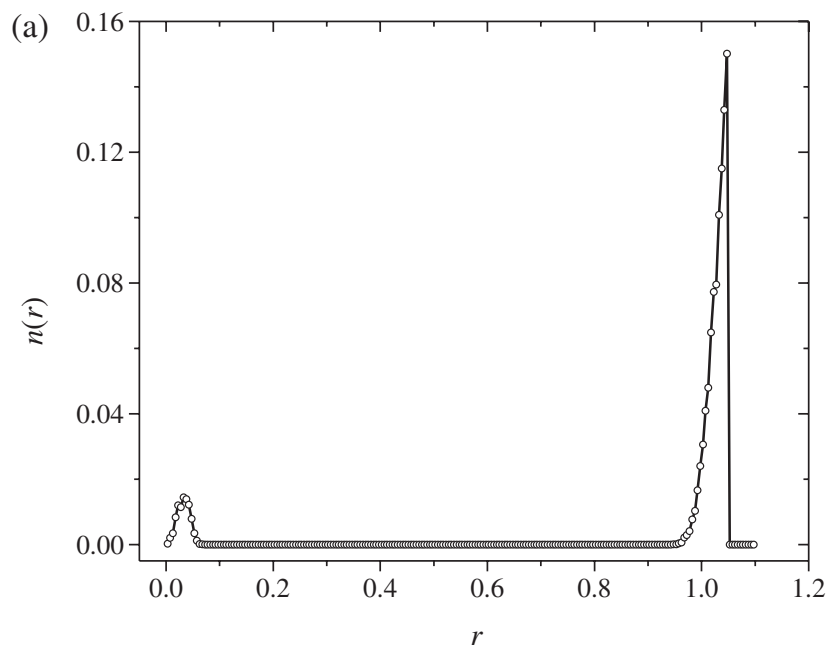

(b)

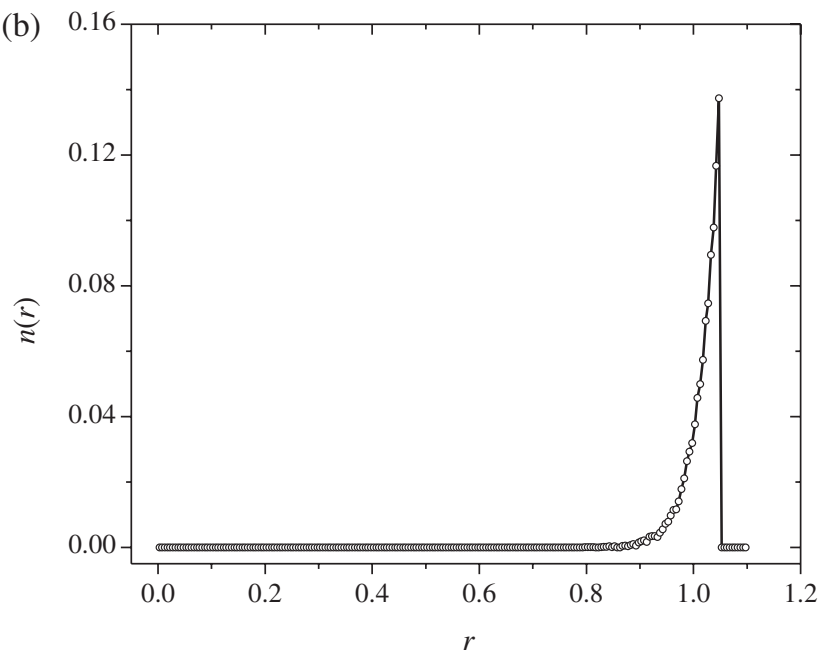

Figure 3. Normalized radial number profiles for $N=11$ and $D / \sigma=2.10$. The (a) 'occupied' and (b) 'vacant' scenario. sphere near the centre of the box this scenario will be referred to as 'occupied'. The profile in figure 3 (b) shows only one peak, indicating that all 11 particles are located in the outer region of the simulation box. The peak in figure 3 (b) is somewhat broadened with respect to the corresponding peak figure 3 (a), due to the absence of a central particle 'pushing' the other particles to the outer region of the simulation box. This scenario will be termed 'vacant'. In our simulations we observe that for a small number of particles the system has a strong preference for the 'vacant' scenario, due to gain of free volume when the spheres are close to a wall. Above $N=10$ the system fills the hole in the middle and the 'occupied' scenario is mostly observed. We are currently determining the exact probabilities of both states.

The existence of an 'occupied' and 'vacant' state in this spherically confined system is already a clear difference compared with rectangularly confined systems $[7,8]$. In these studies the Widom insertion theorem for hard spheres (equation (2)) was used to investigate ordering in small systems. We apply the same Widom insertion strategy to study the possibility of ordering in a system of spherically confined hard spheres. Therefore, we measure the insertion probability $V_{0} / V$ as a function of $N$ and $D / \sigma$, which is shown in figure 4. For both the 'occupied' and 'vacant' state it is observed that at the largest $D / \sigma$ studied (2.30), the insertion probability monotonically decreases with increasing $N$. This points towards the absence of an ordering transition. However, things change already at slightly smaller box sizes. Let us first focus on the 'occupied' scenario (figure 4 (a)). Whereas at $D / \sigma=2.20$ the insertion probability remains roughly constant as $N$ increases from 8 to 9 , an increase of $V_{0} / V$ at this $N$ is observed when the box size is decreased to $D / \sigma=2.15$. Furthermore, another minimum and increase of $V_{0} / V$ is clearly present at respectively $N=11$ and 12 . Upon decreasing box size, both minima and corresponding increases in $V_{0} / V$ at $N=8$ and 11 become more pronounced. However, it must be mentioned that this trend is less clear below $N=10$, where the data are quite noisy. This is a direct consequence of the system being preferentially 'vacant' at these densities, thereby significantly decreasing the statistical accuracy below $N=10$. Nevertheless, the minimum in $V_{0} / V$ at $N=11$ is obvious and indeed becomes more pronounced upon increasing $N$. Similar behaviour is observed for the 'vacant' system at $N=10$ (figure 4(b)). Note that the noise in the data is much smaller compared to the 'occupied' scenario for small $N$.

It is evident that both the 'occupied' and 'vacant' states show an increase of the probability to insert another sphere upon increasing density. This behaviour of $V_{0} / V$ may be suspected to be the result of increasing 

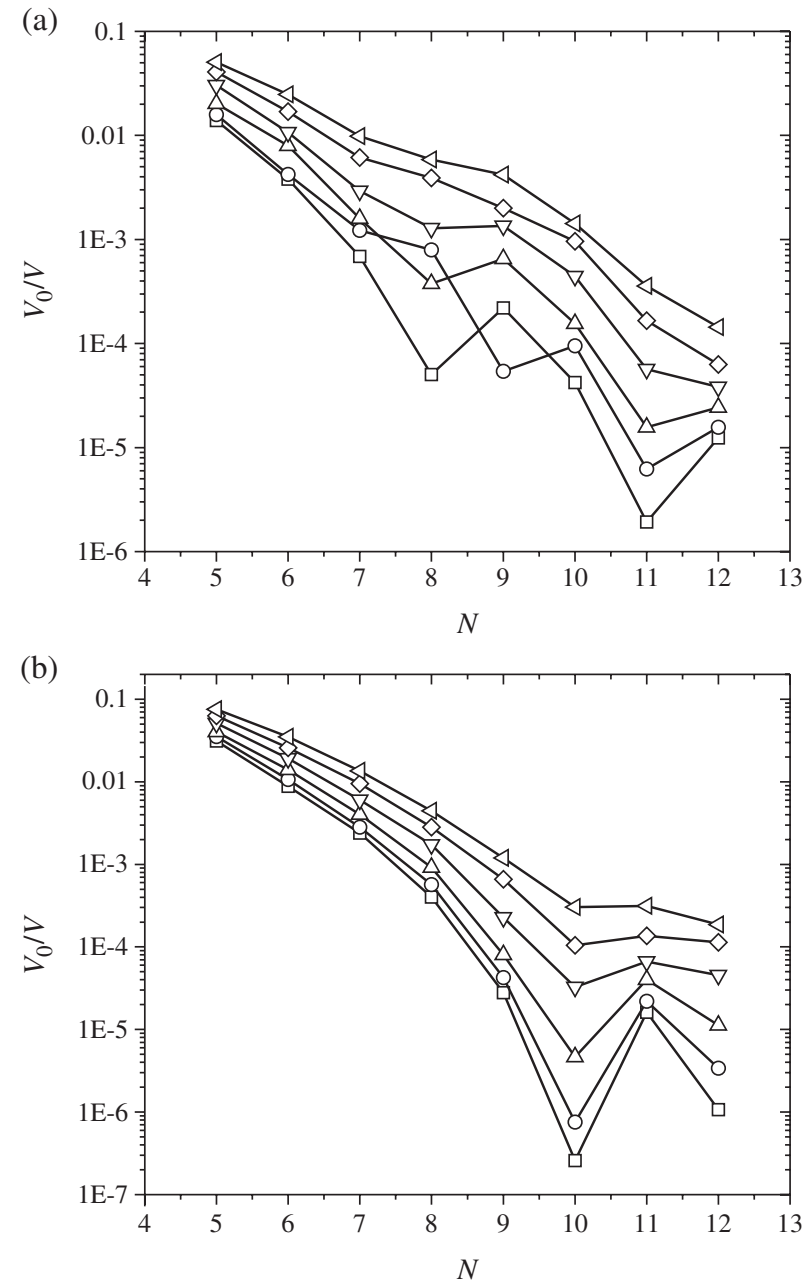

Figure 4. Insertion probability $V_{0} / V$ as a function of $N$ for different $D / \sigma$. The (a) 'occupied' and (b) 'vacant' scenario. Symbols: $D / \sigma=2.10(\square), 2.12(\bigcirc), 2.15(\triangle), 2.20(\nabla), 2.25$ $(\diamond)$ and $2.30(\triangleleft)$.

order in the system. It is interesting to note that the minima in $V_{0} / V$ for both scenarios occur at a different total number of particles, but at an equal number of particles in the coordination shell. This suggests that the structure of the particles constituting the coordination shell is indeed the relevant property to study. To test this notion, we measure the degree of ordering of the particles constituting the coordination shell using the local bond-order parameter as defined by equations (3) and (4) $[13,14]$. Figures 5 (a) and (d) show $q_{6}$ as a function of $N \geq 8$ for respectively the 'occupied' and 'vacant' scenarios. Again, let us first focus on the 'occupied' scenario (figure 5(a)). As $N$ increases from 8 to 9 the local bond-order parameter $q_{6}$ only increases for $D / \sigma=2.10$. However, the increase in $V_{0} / V$ was also observed for $D / \sigma=2.15$ (figure 4(a)). This once more illustrates the statistical uncertainties in the data for the 'occupied' scenario below $N=10$. The increase in $q_{6}$ is most obvious at $N$ increasing from 11 to 12 and gets more pronounced upon decreasing box size. For the 'vacant' scenario (figure 5(d)) $q_{6}$ behaves similar, but now at $N$ increasing from 10 to 11 . The increase of $q_{6}$ seems to be consistent with the increase of the insertion probability. However, also for the largest box size $q_{6}$ increases with increasing $N$, while there is no increase in $V_{0} / V$. Therefore, we also consider the change in $q_{6}$ with $N, \Delta q_{6}$, which is expected to develop a peak in the case of an ordering transition. Figures 5 (b) and (e) show $\Delta q_{6}(N)=q_{6}(N)-q_{6}(N-1)$ for the 'occupied' and 'vacant' scenarios respectively. Indeed we observe that a peak develops in $\Delta q_{6}$ at $N=12$ for the 'occupied' scenario and at $N=11$ for the 'vacant' scenario. The peaks become more pronounced if the box size decreases and are absent if there is no increase in $V_{0} / V$. Note that the increase in $q_{6}$ as well as the peak in $\Delta q_{6}$ are observed at exactly the same $N$ as the increase in $V_{0} / V$. Thus, for both scenarios the behaviour of $q_{6}$ is consistent with an increase of order.

Ordering can also be detected by direct inspection of the simulation snapshots. These are presented in figures 5 (c) and (f) for the 'occupied' ( $N=11$ and 12) and 'vacant' $(N=10$ and 11) scenarios respectively at $D / \sigma=2.10$. The snapshots are oriented such that we are looking along the axis formed by three particles in the case of perfect icosahedral packing. In this orientation the icosahedral structure is clearly recognized since two planes each consisting of 5 particles and mutually twisted by $36^{\circ}$ are visible. In particular, the appearance of these two planes clearly visualizes the formation of the icosahedral structure as $N$ increases from 11 to 12 for the 'occupied' scenario (figure 5(c)) and from 10 to 11 for the 'vacant' scenario (figure 5(f)). Interestingly, in both scenarios a defective icosahedral structure with one ('occupied') or two ('vacant') vacancies is formed, similar to what was reported for rectangularly confined systems [7, 8]. The appearance of a defective solid in spherical confined systems is particularly remarkable due to the absence of corners in the simulation box, in contrast to rectangularly confined systems.

Colloidal dispersions provide a possible route to actually study this behaviour experimentally. A confocal scanning laser microscopy image of spherically confined colloidal hard spheres is presented in figure 6 . The system consists of fluorescently labelled polymethyl methacrylate (PMMA) colloids that are dispersed in decalin in which they interact as hard spheres [15]. By injecting this suspension of PMMA particles into water, an emulsion of decalin droplets in water is formed. As a result, the colloidal particles are trapped in decalin droplets, as shown in figure 6 . Such solvent droplets containing particles may be called 'inverse colloidosomes' [16]. 


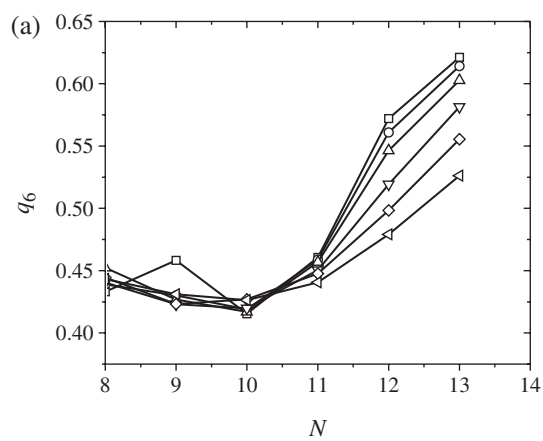

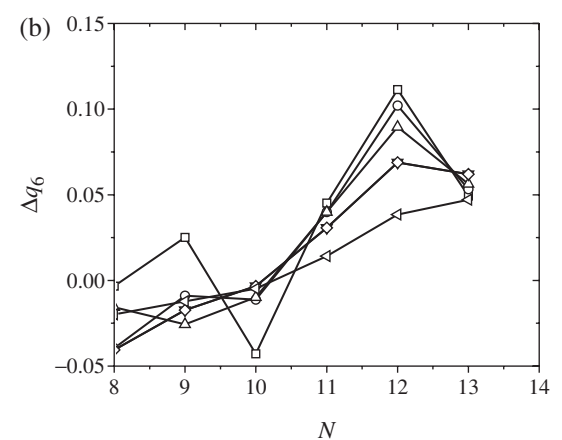

(c)
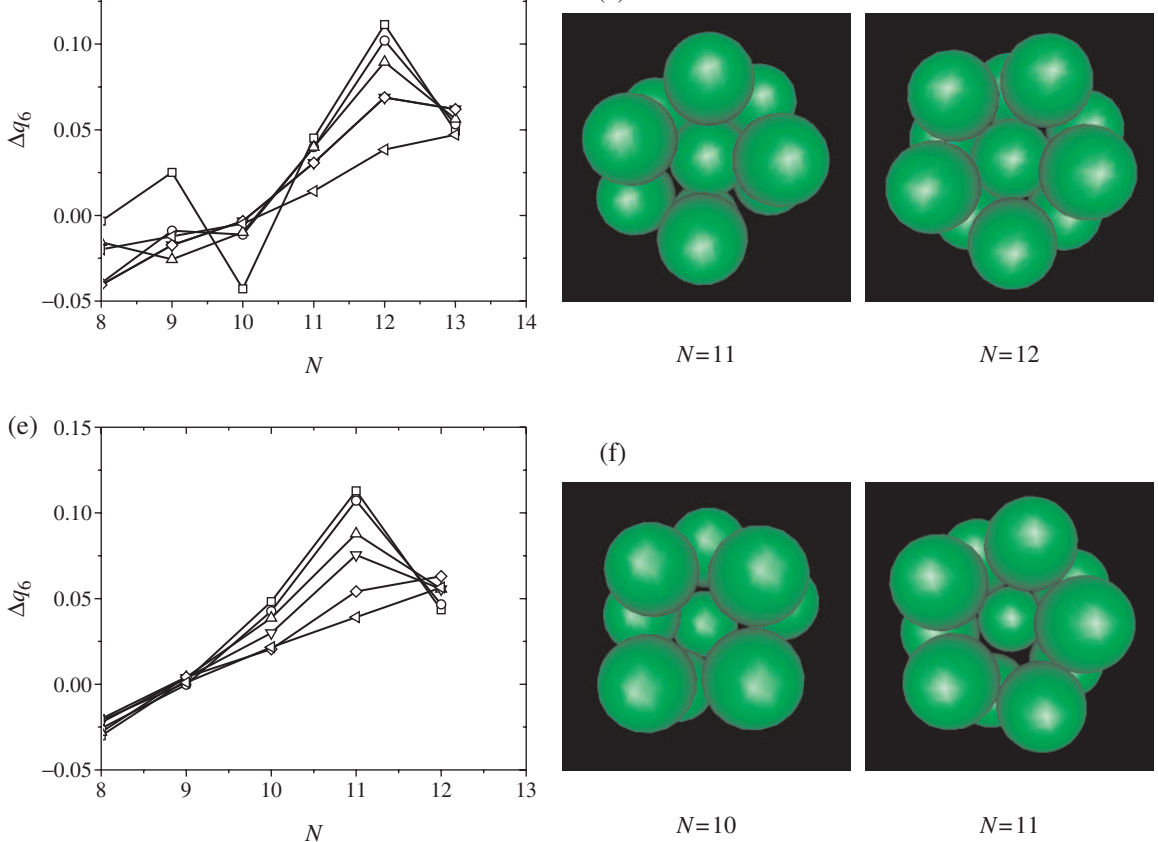

(f)

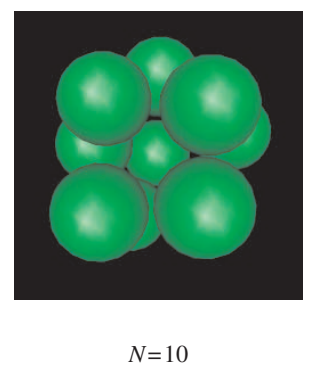

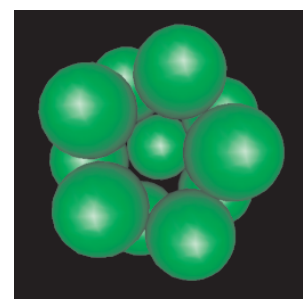

$N=11$

Figure 5. Left and central column: respectively the local bond-order parameter $q_{6}$ and the change in $q_{6}, \Delta q_{6}$ (as defined in the text) as a function of $N$ for different $D / \sigma$ for (a and b) the 'occupied' and (d and e) the 'vacant' scenario. Symbols are the same as in figure 4. Right column: simulation snapshots illustrating the ordering transition for the (c) 'occupied' and (f) 'vacant' scenario at $D / \sigma=2.10$.

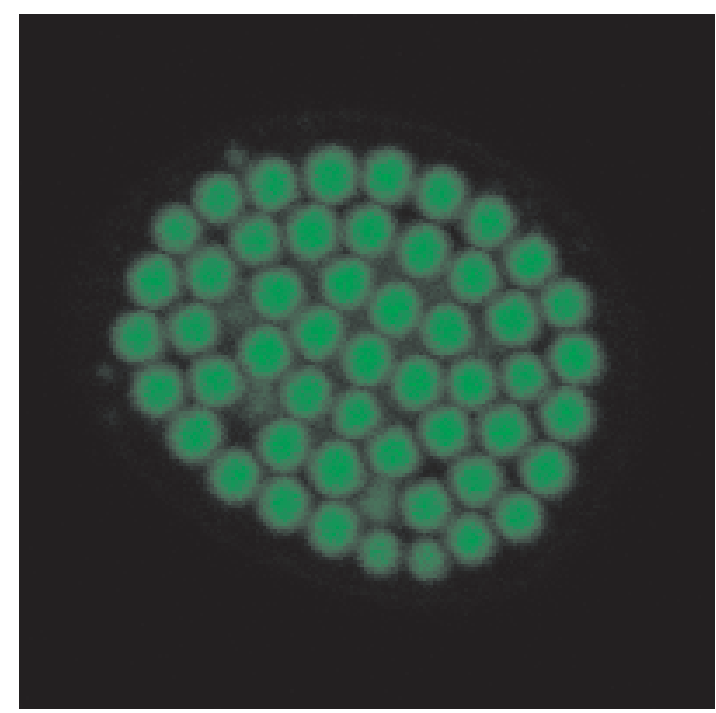

Figure 6. Confocal scanning laser microscopy image of fluorescently labelled PMMA colloids trapped in a droplet of decalin in water. The diameter of the particles is $3.5 \mu \mathrm{m}$ and the field of view is $48 \times 48 \mu \mathrm{m}^{2}$.

The outer particles are confined to the decalin-water interface and thereby establish the confinement of the inner particles, which are free to move thoughout the whole droplet. Controlling the size of the droplets and the number of particles inside, allows a detailed experimental study of small systems of hard spheres in spherical confinement.

\section{Conclusions}

In summary, we have considered a small system of hard spheres confined to a spherical cavity. Using Monte Carlo simulations we obtained the insertion probability $V_{0} / V$ as a function of the number of particles and the box size. Upon decreasing box size an increase of the insertion probability upon increasing number of particles has been observed. A local bond-order analysis demonstrates that such an increase of the insertion probability corresponds to an increase of the order in the system. In particular, the simulation snapshots indicate the formation of defective icosahedral structures. Thus, the Widom particle insertion method is indeed an indicator of the ordering of hard spheres in small spherical cavities.

\section{Acknowledgments}

HNWL would like to thank Ben Widom for many enlightening discussions on the subject of this paper and many other topics. Furthermore, we thank Stephen Williams and Mirjam Leunissen for useful discussions 
and Urs Gasser for the bond-order programs. This work is part of the research programme of the Stichting voor Fundamenteel Onderzoek der Materie (FOM), financially supported by the Nederlandse Organisatie voor Wetenschappelijk Onderzoek (NWO).

\section{References}

[1] B. Widom. J. chem. Phys., 39, 2808 (1963).

[2] J.L. Jackson, L.S. Klein. Phys. Fluids, 7, 228 (1964).

[3] D. Frenkel, B. Smit. Understanding Molecular Simulation. From Algorithms to Applications, 2nd edn., Academic Press, London (2002).

[4] R.P.A. Dullens, D.G.A.L. Aarts, W.K. Kegel. (unpublished).

[5] N.F. Carnahan, K.E. Starling. J. chem. Phys., 51, 635 (1969).
[6] D. Frenkel, A.J.C. Ladd. J. chem. Phys., 81, 3188 (1984).

[7] W.K. Kegel, H. Reiss, H.N.W. Lekkerkerker. Phys. Rev. Lett., 83, 5298 (1999).

[8] W.K. Kegel. Phys. Rev. E, 63, 037104 (2001).

[9] A. González, J.A. White, F. L. Román, S. Velasco, R. Evans. Phys. Rev. Lett., 79, 2466 (1997).

[10] S. Zhou. J. chem. Phys., 110, 2140 (1999).

[11] I.A. Hadjiagapiou. Phys. Rev. E, 65, 021605 (2002).

[12] Z.T. Németh, H. Löwen. Phys. Rev. E, 59, 6824 (1999).

[13] P.J. Steinhardt, D.R. Nelson, M. Ronchetti. Phys. Rev. B, 28, 784 (1983).

[14] P.N. ten Wolde, M.J. Ruiz-Montero, D. Frenkel. J. chem. Phys., 104, 9932 (1996).

[15] G. Bosma, C. Pathmamanoharan, E.H.A. de Hoog, W.K. Kegel, A. van Blaaderen, H.N.W. Lekkerkerker. J. colloid Interface Sci., 245, 292 (2002).

[16] A.D. Dinsmore, M.F. Hsu, M.G. Nikolaides, M. Marquez, A.R. Bausch, D.A. Weitz. Science, 298, 1006 (2002). 